\title{
Genome Medicine: stem cells, genomics and translational research
}

\author{
Stuart H Orkin*
}

Welcome to a new series of articles on stem cell genomics to be published in this and upcoming issues of Genome Medicine. These contributions sample just a few of the many exciting developments in the field of stem cells and genomic research and project ahead to future advances that will make a clear imprint on medicine.

Simply put, stem cells are defined by two properties: self-renewal, the generation of more identical cells, and developmental potency, the capacity to give rise to more differentiated cells. Stem cells come in different 'flavors'. Blood-forming (hematopoietic) stem cells (HSCs), which sustain blood formation through our entire lives, are restricted in their developmental potential to generate only blood cell lineages. On the other hand, embryonic stem (ES) cells can produce the entire repertoire of cells of the body, a property called pluripotency. Although the varieties of stem cells are diverse, it is increasingly evident that fundamental principles and mechanisms underlie self-renewal and differentiation, such that disparate research communities are brought together by common biological themes.

From all the recent excitement in the media about new developments in stem cells, one might think that stem cells were hitherto unknown. Nothing can be further from the truth. Indeed, bone marrow transplantation (BMT), a life-saving procedure for which the Nobel Prize was awarded to E Donnall Thomas in 1990, has been used for more than three decades to treat aplastic anemia and leukemia [1]. Remarkably, BMT was developed empirically through animal and human experimentation before the identification and characterization of HSCs. The extraordinary history of BMT and its clinical development have much to teach us now about how to turn current strategies in the stem cell field into new therapies. For example, although first conceived to treat blood disorders, BMT is gaining momentum in the treatment of non-hematological and non-malignant diseases [2].

*Correspondence: stuart_orkin@dfci.harvard.edu

Dana-Farber Cancer Institute, 450 Brookline Ave., Boston, MA 02116, USA
Current excitement regarding stem cells rests on several converging themes. The derivation of ES cells, first of mouse and then of human origin, provided a platform for the study of pluripotency and the in vitro generation of different cell types. Recent advances in our understanding of the molecular mechanisms underlying lineage determination and differentiation have fueled methods for the interconversion of cells of different lineages. In the most dramatic version of such cellular gymnastics, Shinya Yamanaka and colleagues [3] demonstrated that a small cocktail of regulatory factors can turn virtually any somatic cell into a pluripotent, ES-like cell in a process known as cellular reprogramming. These induced pluripotent stem (iPS) cells have become the focus of brigades of investigators, particularly because they provide a system in which to generate pluripotent cells from an individual of a specific genetic constitution (disease state), giving birth to the notion of 'disease in the dish'.

Improved high-throughput methods, mostly supported by enhanced protein and DNA sequencing platforms, are being used to characterize the cellular proteome, transcriptome, and epigenome (DNA methylation and histone modifications) in comprehensive and exhaustive detail. The vast data from these approaches provide a framework in which to consider the molecular mechanisms underlying cell fate decisions. As cell choice is at the heart of stem cell biology, this knowledge base will guide future research into manipulating normal or disease-related cells for ultimate therapeutic benefit.

This series of articles provides a sense of the breadth and scope of the contemporary stem cell scene. The field touches nearly every corner of medical science. What can we reasonably expect in the coming years? Making predictions is a risky business; nonetheless, I will try. First, the exploitation of 'disease in a dish' should lead to critical new insights into disease processes and stimulate new therapeutic strategies. Second, we should anticipate the development of new therapies based on the stimulation of endogenous stem cells for tissue repair or for reversing the effects of aging, perhaps well before the development of cells as regenerative medicine products. Third, the use of specific cell types produced from human 
pluripotent cells, especially of defined genotypes, should improve the screening of drug toxicity and reduce the overall costs of drug development. Fourth, the connections between epigenetic regulation in pluripotent cells and cancer cells will forge innovative approaches to epigenetic therapy of malignant conditions, and possibly the contribution of epigenetic modulation to non-malignant conditions. Fifth, ex vivo methods for the generation of specific cell types from pluripotent cells or by direct lineage interconversion should drive bioengineering and fuel the next advances in the creation of artificial organs. And finally, with an increased ability to manipulate and generate stem cells of various tissues will come the potential to use gene modification to correct genetic defects or enhance cellular capacities. Such work should stimulate advances in somatic gene therapy of disease.

Although the applications of stem cell biology may be dazzling, it is critical to support fundamental work in the stem cell arena, as only that will ensure a solid foundation for clinical translation and lead to those unexpected discoveries that drive future innovation. We hope that this series on translational stem cell genomics provides a glimpse into this remarkable field.

\section{Abbreviations}

BMT, bone marrow transplantation; ES cells, embryonic stem cells; HSC,

hematopoietic stem cell.

Published: 7 June 2011

\section{References}

1. Thomas ED: Bone marrow transplantation: a review. Semin Hematol 1999, 36:95-103.

2. Smiers FJ, Krishnamurti L, Lucarelli G: Hematopoetic stem cell transplantation for hemoglobinopathies: current practice and emerging trends. Pediatri Clin North Am 2010, 57:181-205.

3. Takahaski K, Yamanaka S: Induction of pluripotent stem cells from mouse embryonic and adult fibroblast cultures by defined factors. Cell 2006 126:663-676.

doi:10.1186/gm250

Cite this article as: Orkin SH: Genome Medicine: stem cells, genomics and translational research. Genome Medicine 2011, 3:35. 